\title{
Diagnostic Biomarkers - Exploring the Potential of Cortisol and Yawning in the Detection of Neurological Disease Processes
}

\author{
Simon B.N. Thompson ${ }^{1,2,3}$ \\ ${ }^{1}$ Faculty of Science and Technology, Bournemouth University, BH15 1YE, UK \\ ${ }^{2}$ Dementia Institute, Bournemouth University, BH1 3LT, UK \\ ${ }^{3}$ International Scientific Council for Research into Multiple Sclerosis, Université Paris X Ouest Nanterre La Défense, 92000 Nanterre, France
}

Corresponding author: Simon B.N. Thompson, Faculty of Science and Technology, Bournemouth University, Poole House (P-305), Poole, BH12 5BB, UK, Tel: +44 1202 961558; E-mail: simont@bournemouth.ac.uk

Received: May 25, 2016; Accepted: Jun 09, 2016; Published: Jun 13, 2016

\begin{abstract}
Yawning continues to pose as a scientist's conundrum. Evidence is presented of yawning and contagious yawning in a number of different neurological disorders. Explanations are discussed in the context of disparate neurological disorders together with proposals for how these findings may be linked. Thus, greater understanding of yawning and of neurological disorders may be achieved by exploring common neuro-chemical pathways and the involvement of neurotransmitters that are implicated in these different disorders. Finally, contagious yawning is discussed in the context of the susceptibility of persons and the similarity this presents with our understanding of the mechanisms involved in hypnosis.
\end{abstract}

Keywords: Neurological disease; Diagnostic biomarkers; Neurotransmitters; Neuro-chemical pathways

\section{Introduction}

The term 'biological marker' or 'biomarker' dates back to 1980 (Aronson, 2005) and is defined as an entity that measures a particular biological state, objectively measured and evaluated as an indicator of normal biological processes, pathogenic processes or pharmacological responses to a therapeutic intervention [1].

Historically, a large number of people are selected from a given disease category to identify the biomarker. Following this 'proof of concept', experimental validation takes place using various methods, for example, histology, and then followed by analytical validation exploring several parameters: sensitivity; specificity; robustness; accuracy; and reproducibility [2].

Cortisol is a naturally occurring hormone that is considered important for immune protection and stress regulation [3-5] (Figure 1). Measuring the levels of cortisol is useful for monitoring a number of different medical conditions including cases where high levels of stress are known such as adrenal insufficiency, or Cushing's disease, and for certain neurological diseases, including multiple sclerosis [6].
The link between assay levels obtained via invasive blood collection and non-invasive, saliva sampling is directly proportional to the serum unbound cortisol concentration both in normal women and men [7].

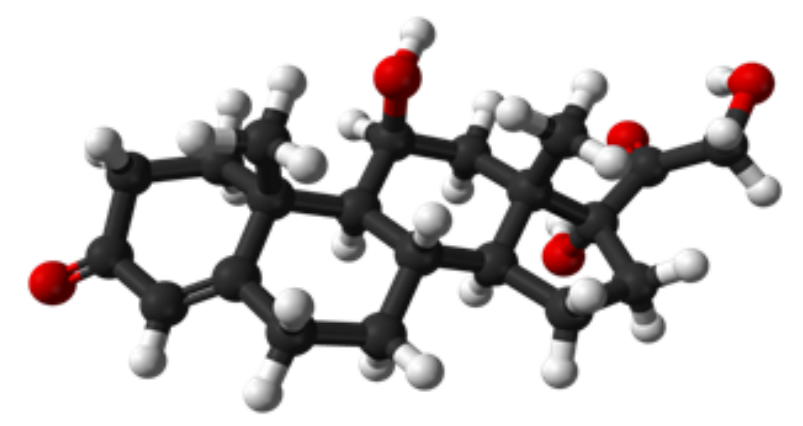

Figure 1 Cortisol hormone showing atoms and bonds.

A greater level of neuromuscular fatigue and larger responses in serum hormone concentrations has been evidenced after hypertrophic variable resistance loadings [8]. This has led to identifying markers of fatigue [9]; in particular, following post-match professional rugby [10] and also in young elite athletes [11], and in elite tennis players [12].

Tiredness due to physical exercise and even mental concentration on tasks is often referred to as 'fatigue' and is sometimes associated with yawning. The Thompson Cortisol Hypothesis [13] is the first evidence-based report that links cortisol, with yawning, and demonstrates that cortisol rises when we yawn. A common symptom of multiple sclerosis (MS) is fatigue [14] which is also associated with excessive yawning and a rise in brain temperature, governed by the hypothalamus [15].

Temperature regulation and circadian rhythm is the responsibility of the hypothalamus which is intimately linked to two other body structures, the pituitary gland and the adrenal glands which secrete adrenaline. Typically, the hypothalamus-pituitary-adrenal (HPA) axis produces sufficient hormones to protect against stress and ensures the body is ready for physical activity. 
Produced by the zona fasciculate of the adrenal cortex [16], the rise in cortisol level possibly triggers the response to yawn and in turn, lowers the temperature of the brain [17-19].

The author's team at Bournemouth University and the French teams at Amiens University Hospital and the University of Picardy Jules Verne [20] tested the theory that intrinsic and phasic fatigue may be associated with cortisol levels and that yawning, known to be associated with elevated cortisol levels [6], may occur in association with fatigue. This could explain why people with multiple sclerosis yawn when they are frequently fatigued.

The role of cortisol in mental and motor tasks together with the recruitment of brain regions during these tasks has been explored in a recent fMRI study [20]. In healthy participants, cortisol levels were found to be higher and had greater reduction in levels during mental versus motor tasks. These findings also showed that recruitment of brain-stem and hypothalamus regions, which are considered to be important in cortisol activity, were affected to differing extents.

For example, at low cortisol levels, mental task participants had less activity in the regions than their physical task counterparts and when cortisol levels were higher, wider spread recruitment of these brain regions was seen in the mental task participants. For the physical task participants, the spread was at comparative low levels of cortisol.

These encouraging and intriguing results are yet to be seen in people with immune-suppression, neurological disease, and cortisol insufficiency. However, if cortisol is implicated in these brain regions, this tends to lend support for the Thompson Cortisol Hypothesis. Furthermore, it suggests that brain region recruitment is likely to be dependent upon cortisol levels as well as perception of stress in a task. It is suggested that the mental tasks in this study were perceived as being more stressful than the physical tasks but demanded higher cortisol levels to promote wider spread brain region activity.

Cortisol, together with fatigue-related yawning may provide scientists and neurologists with a new biomarker of underlying neurological disease. As with any potential and new biomarkers, the systematic evaluation of healthy and nonhealthy participants needs to be carried out but it does present us with considerable new possibilities for the detection, and perhaps early intervention of multiple sclerosis in the near future.

\section{References}

1. Strimbu K, Jorge T (2010) What are biomarkers? Current Opinion in HIV and AIDS 6: 463-466.

2. Mansfield E (2012) In vitro diagnostics - role in efficacy biomarker assessment. Retrived from http://www.fda.gov/ downloads/Drugs/NewsEvents/UCM 3007 31.pdf.
3. Thompson SBN (2010) The dawn of the yawn: is yawning a warning? Linking neurological disorders. Med Hyp 75: 630-633.

4. Thompson SBN (2015) Yawning and cortisol as a potential biomarker for early detection of multiple sclerosis. International Journal of Medical, Health, Biomedical \& Pharmaceutical Engineering 9: 358-362.

5. Thompson SBN (2015) Health psychology intervention identifying early symptoms in neurological disorders. Int J Med Health Biomed Bioeng Pharma Eng 4: 351-355.

6. Thompson SBN, Bishop P (2012) Born to yawn? Understanding yawning as a warning of the rise in cortisol levels: randomized trial. Interact J Med Res e4: 1-9.

7. Vining RF, McGinley RA, Maksvytis JJ, Ho KY (1983) Salivary cortisol: a better measure of adrenal cortisol function than serum cortisol. Ann Clin Biochem 6: 329-335.

8. Walker S, Taipale RS, Nyman K, Kraemer WJ, Hakkinen K (2011) Neuromuscular and hormonal responses to constant and variable resistance loadings. Med Sci Sports Exer 1: 26-33.

9. Bresciani G, Cuevas MJ, Molinero O, Almar M, Suay F, et al. (2011) Signs of overload after an intensified training. Int J Sports Med 5: 338-343.

10. McLellan CP, Lovell DI, Gass GC (2011) Markers of post-match fatigue in professional rugby league players. J Strength Condit Res 4: 1030-1039.

11. Locke S, Osborne M, O'Rourke P (2011) Persistent fatigue in young athletes: measuring the clinical recovery and identifying variables affecting clinical recovery. Scandin J Med Sci Sports 1: 90-97.

12. Gomes RV, Coutts AJ, Viveiros L, Aoki MS (2011) Physiological demands of match-play in elite tennis: a case study. Europ J Sport Sci 2: 105-109.

13. Thompson SBN (2014) Yawning, fatigue, and cortisol: expanding the Thompson Cortisol Hypothesis. Med Hyp 4: 494-496.

14. Fleming WE, Pollak CP (2005) Sleep disorders in multiple sclerosis. Sem Neurol 25: 64-68.

15. Gallup AC, Gallup JrGG (2007) Yawning as a brain cooling mechanism: Nasal breathing and forehead cooling diminish the incidence of contagious yawning. Evol Psychol 1: 92-101.

16. Schillings WJ (2008) Physiology and tests of adrenal cortisol function. Glob Lib Wom Med.

17. Thompson SBN (2015) Pathways to yawning: making sense of the Thompson Cortisol Hypothesis. Med Res Arch 3: 1-7.

18. Thompson SBN, Richer S (2015) How yawning and cortisol regulates the attentional network. J Neurosci Rehab 1: 1-9.

19. Thompson SBN, Daly S, Le Blanche A, Adibi M, Belkhira C, et al. (2016) fMRI randomized study of mental and motor task performance and cortisol levels to potentiate cortisol as a new diagnostic biomarker. J Neurol Neurosci 7: 92.

20. Périn B, Gogefroy O, Fall S, de Marco G (2010) Alertness in young healthy subjects: an $\mathrm{FMRI}$ study of brain region interactivity enhanced by a warning signal. Brain Cog 72: 271-281. 